\section{Kidney \\ Blood Pressure Research}

Kidney Blood Press Res 2017;42:575-586

DOI: 10.1159/000480674

Published online: September 18, 2017

This article is licensed under the Creative Commons Attribution-NonCommercial-NoDerivatives 4.0 International License (CC BY-NC-ND) (http://www.karger.com/Services/OpenAccessLicense). Usage and distribution tional License (CC BY-NC-ND) (http://www.karger.com/Services/OpenAccessLicense). Usage and dist
for commercial purposes as well as any distribution of modified material requires written permission.

\title{
The Clinical Significance of Physical Activity in Maintenance Dialysis Patients
}

\author{
Seok Hui Kang ${ }^{\mathrm{a}}$ Jun Young Do ${ }^{\mathrm{a}}$ Hye Yun Jeong ${ }^{\mathrm{b}}$ So-Young Lee ${ }^{\mathrm{b}}$ Jun Chul Kim \\ aDivision of Nephrology, Department of Internal Medicine, Yeungnam University Hospital, Daegu, \\ Republic of Korea, 'Division of Nephrology, Department of Internal Medicine, CHA Bundang Medical \\ Center, CHA University School of Medicine, Seongnam, Republic of Korea, 'Division of Nephrology, \\ Department of Internal Medicine, CHA Gumi Medical Center, CHA University, Gumi, Republic of Korea
}

\section{Key Words}

Physical activity - Quality of life - Peritoneal dialysis - Hemodialysis • Frailty • Disability • Exhaustion

\begin{abstract}
Background: The aim of the present study was to evaluate the effects of physical activity on various aspects in Asian dialysis patients. Methods: This was a retrospective cohort study. Study participants were recruited from 27 hospitals or dialysis centers in Korea $(n=1611)$. The participants were divided into 3 groups according to the degree of regular exercise: Inactive group, Intermediate group, and Active group. Results: The proportions of patients with frailty and the presence of each component decreased as physical activity increased. The presence and numbers of disabilities decreased as physical activity increased. The number of participants with a history of fall during the last 12 months was $149(20.5 \%)$ in the Inactive group, $88(16.9 \%)$ in the Intermediate group, and 48 (13.2\%) in the Active group. Physical component scale and mental component scale scores increased as physical activity increased. The survival rate for all-cause death at 500 days was $95.5 \%$ in the Active group, $95.2 \%$ in the Intermediate group, and $93.5 \%$ in the Inactive group. Conclusion: High physical activity was associated with favorable results for most health-related quality of life scale scores, including frailty, disability, and exhaustion, in Korean dialysis patients.
\end{abstract}




\section{Kidney Blood Pressure Research}

\section{Introduction}

Dialysis patients are particularly susceptible to decreased health-related quality of life (HRQoL) or physical performance compared with patients who undergo kidney transplantation [1-4]. Therefore, many studies have focused on these issues in dialysis patients. Previous studies showed that various factors including anemia, malnutrition, and metabolic disturbances are associated with HRQoL or physical performance; however, there has not been much improvement in these problems [6-8]. Further investigations of new risk factors or proper interventions are needed to improve HRQoL or physical performance in dialysis patients.

Physical activity is a well-known factor that improves HRQoL, physical performance, and other clinical outcomes in the general population [9]. Physical inactivity is associated with an imbalance of energy expenditure that can increase comorbidities, such as hypertension, diabetes mellitus (DM), coronary artery disease (CAD), and depressive mood, which can aggravate such issues [9]. Considering these associations in the general population, physical activity in patients with chronic kidney disease (CKD) can also be associated with improvements in these issues. In addition, Kielstein et al. investigated the association between physical activity and endogenous nitric oxide inhibitor asymmetric dimethylarginine (ADMA) as a uremic toxin even without comorbidities [10]. They showed that ADMA infusion alone without CKD significantly decreased brain-derived neutrophic factors and physical activity. These reveal that patients with CKD are inherently at risk of decreased physical activity regardless of various comorbidities.

Barcellos et al. reviewed the effects of exercise in patients in various CKD stages [11]. For dialysis patients, exercise significantly improved oxygen peak consumption and strength. The association between exercise and C-reactive protein (CRP) level or HRQoL was not consistent. For non-dialysis patients, exercise significantly improved oxygen peak consumption and HRQoL. However, the association between exercise and CRP level or progression of CKD was also not consistent. Most included studies enrolled only dialysis patients. The effect of exercise in dialysis patients had relatively strong evidences, but not in pre-dialysis patients.

Previous studies showed favorable effects of physical activity in dialysis patients; however, these have mainly focused on Western populations or mortality only [12-23]. Few studies have comprehensively assessed the effects of physical activity on various factors, including HRQoL, frailty, disability, exhaustion, and mortality, in Asian populations. The aim of the present study was to evaluate the effects of physical activity on these various aspects in Asian dialysis patients.

\section{Patients and Methods}

\section{Study population}

The study participants were initially enrolled in a previous study [24]. Briefly, study participants were recruited from 27 hospitals or dialysis centers in Daegu/Kyungsangpook-do between July and December 2012. A total of 2737 participants who underwent dialysis were included. Participants were excluded from the present study if they met the following criteria: age $<20$ years $(n=12)$, hospitalization during the previous 3 months except for vascular access problems $(\mathrm{n}=351)$, dialysis duration < 6 months $(\mathrm{n}=164)$, inability to walk with or without an assistive device $(n=79)$, lack of laboratory findings $(n=117)$, refusal to participate $(n=254)$, or inability to communicate with interviewers $(n=149)$. As a result, 1611 participants were ultimately included in this study. There were no dialysis units to encourage exercise during the dialysis session. The hospital's ethics committee approved the study protocol, and the study was conducted in accordance with the principles originating from the Declaration of Helsinki. 


\section{Kidney Blood Pressure Research}

Kang et al.: Physical Activity in Dialysis Patients

\section{Study variables}

Demographic and laboratory data collected at enrollment included the following: age, sex, body mass index (BMI; $\mathrm{kg} / \mathrm{m}^{2}$ ), DM, CAD, cerebrovascular disease (CVD), dialysis vintage (years), education level, and levels of hemoglobin $(\mathrm{mg} / \mathrm{dL})$, blood urea nitrogen (BUN; mg/dL), creatinine $(\mathrm{mg} / \mathrm{dL})$, calcium $(\mathrm{mg} / \mathrm{dL})$, phosphorus (mg/dL), total cholesterol (mg/dL), intact-parathyroid hormone (i-PTH; pg/mL), and highsensitivity CRP (hs-CRP; mg/dL). DM was defined as a self-reported history and medical record of DM diagnosis or a fasting glucose level $\geq 126 \mathrm{mg} / \mathrm{dL}$. CAD was defined as a self-reported history and medical record of angina, myocardial infarction, or congestive heart failure. CVD was defined as a self-reported history and medical record of stroke. All-cause and cardiovascular mortalities up to December 2014 were retrieved from the patients' medical records.

Assessment of HRQoL scale scores

HRQoL was assessed by the Kidney Disease Quality of Life (KDQOL)-SF ${ }^{\mathrm{TM}} 1.3$ Korean version [25]. KDQOL- SF ${ }^{\mathrm{TM}} 1.3$ includes the SF-36 scale (36 items) and kidney disease-specific scale (11 items). The SF-36 includes the following 8 domains: physical functioning (PF), role limitations due to physical health problems (RP), body pain (BP), general health (GH), vitality (VT), social functioning (SF), role limitations due to emotional problems (RE), mental health (MH), and overall health rating (OHR). A total score of 0-100 is calculated for each domain. A low score indicates a low quality of life. These scales have been used to calculate the physical component scale (PCS) and mental component scale (MCS) [26, 27]. The 11 kidney disease-specific items consist of symptom/problem, kidney disease effects, kidney disease burden, work status, cognitive function, quality of social interaction (QSI), sexual function, sleep, social support, patient satisfaction, and dialysis staff encouragement. Questionnaires were completed during the dialysis session.

\section{Definitions}

We evaluated disability using 4 questions regarding activities of daily living (ADLs) to assess whether the participants currently needed help from another person to have a meal, dress/undress, get in or out of bed, or take a bath or shower. For each question, they answered one of 3 responses: no help needed, some help needed, or complete help needed. Disability was defined as the inability to perform at least one of the 4-ADL domains without help [28].

Exhaustion was measured using 2 questions from the Center for Epidemiological Studies Depression Scale [29]: (1) I felt that everything I did was an effort; and (2) I could not get going. The question asked was: "How often did you feel this way?" The answer was rarely or none of the time, some or a little of the time, a moderate amount of the time, or most of the time. The participants answering moderate or most of the time to either or both of the 2 questions were identified as having exhaustion. A fall was defined as an event that resulted in a participant coming to rest unintentionally on the ground or a lower level with or without losing consciousness during the last 12 months. Serious falls were defined as head injury requiring hospitalization, joint dislocations, severe sprains, or a laceration requiring sutures [30].

Physical activity was defined as the presence of regular exercise during leisure time for the past 3 months. The participants were divided into 3 groups according to exercise amount. The Active group was defined as engaging in moderate activity for $>30 \mathrm{~min} /$ day for 5 days a week or at a high intensity for $>20 \mathrm{~min} /$ day for 3 days a week [9]. The Inactive group was defined as engaging in physical activity $<1$ time per week during their leisure time for the previous 3 months. The Intermediate group was defined as participants whose activity levels were between those of the Inactive and Active groups. Most of the participants in the Inactive group provided the following causes of their low physical activity: pain, fatigue, lack of will to exercise, depressive mood, lack of time to exercise, unsure how to exercise, unsure about the importance of exercise, lack of equipment or place to exercise, and fear of injury. Participants answered nonopen questions and chose from multiple choices.

Frailty was defined using modified criteria previously described elsewhere [31]. Components included slowness, poor endurance, physical inactivity, and unintentional weight loss. Slowness and poor endurance were determined by the PF scale of the SF-36 (2 points for PF scale < 75) and the VT scale of the SF-36 (1 point for VT scale < 55), respectively. Participants who were physically inactive were classified into the Inactive group (1 point for physical inactivity). Unintentional weight loss was defined as unintentional body weight loss $>4.5 \mathrm{~kg}$ or $5 \%$ of the baseline value over the past year (1 point for weight loss). All points of 


\section{Kidney \\ Blood Pressure Research}

Table 1. Participants' clinical characteristics

\begin{tabular}{|c|c|c|c|c|}
\hline & Inactive $(\mathrm{n}=728)$ & Intermediate $(\mathrm{n}=520)$ & Active $(n=363)$ & $P$-value \\
\hline Age (years) & $55.0 \pm 14.0$ & $56.1 \pm 12.2$ & $57.3 \pm 11.6^{*}$ & 0.021 \\
\hline Sex (male, \%) & $378(51.9 \%)$ & $282(54.2 \%)$ & $240(66.1 \%)$ & 0.000 \\
\hline Body mass index $\left(\mathrm{kg} / \mathrm{m}^{2}\right)$ & $22.5 \pm 3.5$ & $22.2 \pm 3.0$ & $22.2 \pm 2.9$ & 0.155 \\
\hline Diabetes mellitus (\%) & $274(37.6 \%)$ & $209(40.2 \%)$ & $154(42.4 \%)$ & 0.293 \\
\hline Coronary artery disease (\%) & $120(16.5 \%)$ & $75(14.4 \%)$ & $59(16.3 \%)$ & 0.591 \\
\hline Cerebrovascular disease (\%) & $58(8.0 \%)$ & $49(9.4 \%)$ & $37(10.2 \%)$ & 0.428 \\
\hline Dialysis vintage (years) & $5.4 \pm 4.8$ & $5.1 \pm 4.3$ & $4.8 \pm 4.1$ & 0.118 \\
\hline Education level & & & & 0.050 \\
\hline$\leq 6^{\text {th }}$ grade & $180(24.7 \%)$ & $97(18.7 \%)$ & $76(20.6 \%)$ & \\
\hline $7^{\text {th }}-12^{\text {th }}$ grade & $142(19.5 \%)$ & $96(18.5 \%)$ & $80(22.0 \%)$ & \\
\hline$>12^{\text {th }}$ grade & $406(55.8 \%)$ & $327(62.9 \%)$ & $207(57.0 \%)$ & \\
\hline Hemoglobin $(\mathrm{mg} / \mathrm{dL})$ & $10.4 \pm 1.0$ & $10.5 \pm 0.9$ & $10.5 \pm 0.8$ & 0.665 \\
\hline Serum albumin $(\mathrm{g} / \mathrm{dL})$ & $3.9 \pm 0.4$ & $3.9 \pm 0.4$ & $3.8 \pm 0.4$ & 0.073 \\
\hline Blood urea nitrogen $(\mathrm{mg} / \mathrm{dL})$ & $56.6 \pm 15.3$ & $60.0 \pm 14.6$ & $61.0 \pm 15.1$ & 0.329 \\
\hline Creatinine $(\mathrm{mg} / \mathrm{dL})$ & $10.4 \pm 2.9$ & $10.5 \pm 3.1$ & $10.6 \pm 3.0$ & 0.515 \\
\hline Calcium $(\mathrm{mg} / \mathrm{dL})$ & $8.7 \pm 0.8$ & $8.7 \pm 0.8$ & $8.7 \pm 0.8$ & 0.512 \\
\hline Phosphorus (mg/dL) & $5.4 \pm 1.4$ & $5.3 \pm 1.3$ & $5.2 \pm 1.3$ & 0.181 \\
\hline Total cholesterol (mg/dL) & $154.5 \pm 36.5$ & $153.6 \pm 36.9$ & $153.6 \pm 39.3$ & 0.886 \\
\hline Intact parathyroid hormone $(\mathrm{pg} / \mathrm{mL})$ & $314.5 \pm 424.7$ & $255.7 \pm 271.2 *$ & $234.0 \pm 220.9 *$ & 0.000 \\
\hline Dialysis modality (hemodialysis, \%) & $567(77.9 \%)$ & $414(79.6 \%)$ & $266(73.3 \%)$ & 0.079 \\
\hline High sensitivity C-reactive protein $(\mathrm{mg} / \mathrm{dL})$ & $0.73 \pm 1.39$ & $0.72 \pm 2.08$ & $0.54 \pm 1.36$ & 0.397 \\
\hline
\end{tabular}

scoring of each frailty component were summed. Those participants with $\geq 3$ points, 1 or 2 points, and 0 points were defined as having frailty, pre-frailty, and non-frailty, respectively.

\section{Statistical analyses}

The data were analyzed using the statistical software SPSS version 21 (Chicago, IL, USA). Categorical variables were expressed as both counts and percentages. Continuous variables were expressed as mean \pm standard deviation (SD) or mean \pm standard error (SE). The Pearson's $\chi^{2}$ or Fisher's exact test was used to analyze categorical variables. For continuous variables, means were compared using the $t$-test or analysis of variance, followed by a post-hoc Tukey comparison. The survival estimates were calculated using KaplanMeier curves. Multivariate analysis was performed using analysis of covariance. For analysis of covariance, the covariates were age, sex, BMI, education level, dialysis vintage, DM, CVD, CAD, serum albumin level, i-PTH level, and dialysis modality. The level of statistical significance was set at $P<0.05$.

\section{Results}

\section{Participants' clinical characteristics}

The number of participants in the Inactive, Intermediate, and Active groups was 728, 520 , and 363, respectively (Table 1). The numbers of participants with hemodialysis (HD) and peritoneal dialysis (PD) were 364 (22.6\%) and 1247 (77.4\%), respectively. Among the HD patients, the numbers of participants who underwent 2 and 3 HD sessions/week were 77 (6.2\%) and 1170 (93.8\%), respectively. The numbers of participants who underwent 2 HD sessions/week were $29(5.1 \%)$ in the Inactive group, 29 (7.0\%) in the Intermediate group, and $19(7.1 \%)$ in the Active group $(P=0.464)$. The most common dialysis modality in our study was HD and most of these participants underwent 3 HD sessions/week. There were no significant differences in dialysis modality or frequency of HD session among the 3 groups.

In the patients in the Inactive group, the most common cause of physical inactivity was unwillingness to exercise (302 participants, 41.5\%). The numbers of participants who cited fatigue, lack of time to exercise, pain, unsure how to exercise, depressive mood, fear of injury, lack of equipment or place to exercise, and unsure about the importance of exercise were 287 (39.4\%), 186 (25.6\%), 185 (25.4\%), 72 (9.9\%), 62 (8.5\%), 49 (6.7\%), 41 (5.6\%), and 24 


\section{Kidney Blood Pressure Research}

(3.3\%), respectively. We have divided the participants in the Inactive group into those with unwilling nes s only ( $\mathrm{n}=130$ ) and others (n $=$ 598). Comparison of clinical characteristics is shown in Table 2. Participants with unwillingness only had a younger age and higher education levels than those in others.
Table 2. Clinical characteristics according to the cause of physical inactivity

\begin{tabular}{lccc}
\hline & $\begin{array}{c}\text { Unwillingness } \\
\text { only }(\mathrm{n}=130)\end{array}$ & $\begin{array}{c}\text { Others } \\
(\mathrm{n}=598)\end{array}$ & $P$-value \\
\hline Age (years) & $50.4 \pm 12.4$ & $56.0 \pm 14.1$ & 0.000 \\
Sex (male, \%) & $66(50.8 \%)$ & $312(52.2 \%)$ & 0.423 \\
Body mass index (kg/m²) & $22.3 \pm 3.1$ & $22.6 \pm 3.6$ & 0.260 \\
Diabetes mellitus (\%) & $48(36.9 \%)$ & $226(37.8 \%)$ & 0.468 \\
Coronary artery disease (\%) & $16(12.3 \%)$ & $104(17.4 \%)$ & 0.097 \\
Cerebrovascular disease (\%) & $7(5.4 \%)$ & $51(8.5 \%)$ & 0.153 \\
Dialysis vintage (years) & $5.4 \pm 5.5$ & $5.4 \pm 4.6$ & 0.972 \\
Education level & & & 0.001 \\
$\leq 6^{\text {th }}$ grade & $18(13.8 \%)$ & $162(27.1 \%)$ & \\
$7^{\text {th }}$ - 12th grade & $22(16.9 \%)$ & $120(20.1 \%)$ & \\
$>12^{\text {th }}$ grade & $90(69.2 \%)$ & $316(52.8 \%)$ & \\
Hemoglobin (mg/dL) & $10.4 \pm 0.9$ & $10.5 \pm 1.0$ & 0.533 \\
Serum albumin (g/dL) & $3.9 \pm 0.4$ & $3.9 \pm 0.4$ & 0.894 \\
Blood urea nitrogen (mg/dL) & $58.2 \pm 13.8$ & $59.9 \pm 15.6$ & 0.235 \\
Creatinine (mg/dL) & $10.5 \pm 3.0$ & $10.3 \pm 2.9$ & 0.524 \\
Calcium (mg/dL) & $8.8 \pm 0.8$ & $8.7 \pm 0.9$ & 0.607 \\
Phosphorus (mg/dL) & $5.3 \pm 1.1$ & $5.4 \pm 1.4$ & 0.658 \\
Total cholesterol (mg/dL) & $153.7 \pm 35.2$ & $154.7 \pm 36.8$ & 0.762 \\
Intact parathyroid hormone (pg/mL) & $322.0 \pm 533.3$ & $312.9 \pm 397.7$ & 0.854 \\
Dialysis modality (hemodialysis, \%) & $96(73.8 \%)$ & $471(78.8 \%)$ & 0.135 \\
High sensitivity C-reactive protein (mg/dL) & $0.82 \pm 1.53$ & $0.70 \pm 1.35$ & 0.561 \\
\hline Data arexpress & & \\
\hline
\end{tabular}

Data are expressed as numbers (percentages) for categorical variables and mean \pm standard deviations for continuous variables. $P$-values were tested by the $t$-test for continuous variables and Pearson's $\chi^{2}$ or Fisher's exact tests for categorical variables.

Comparison of frailty and disability by physical activity level

The numbers of participants with pre-frailty and frailty were $343(47.1 \%)$ and 384 $(52.7 \%)$ in the Inactive group; $246(47.3 \%)$ and 119 (22.9\%) in the Intermediate group; and $152(41.9 \%)$ and $54(14.9 \%)$ in the Active group, respectively $(P<0.001)$. For each frailty component, the numbers of participants with slowness, poor endurance, and unintentional weight loss were $348(47.8 \%), 560(76.9 \%)$, and $91(12.5 \%)$ in the Inactive group; 147 (28.3\%), $325(62.5 \%)$, and $42(8.1 \%)$ in the Intermediate group; and $65(17.9 \%), 182$ (50.1\%), and $32(8.8 \%)$ in the Active group, respectively $(P<0.001$ for slowness, $P<0.001$ for poor endurance, and $P=0.024$ for unintentional weight loss). The proportions of patients with frailty and the presence of each component decreased as physical activity increased.

The proportions of participants with disability in the Inactive, Intermediate, and Active groups were 171 (23.5\%), 93 (17.9\%), and 40 (11.0\%), respectively (Fig. 1A; $P<0.001$ ). The mean numbers of disabilities (means $\pm \mathrm{SE}$ ) were $0.46 \pm 0.04$ in the Inactive group, $0.33 \pm 0.04$ in the Intermediate group, and $0.18 \pm 0.03$ in the Active group $(P<0.001)$. The multivariate analysis showed similar results $(0.48 \pm 0.03$ in the Inactive group, $0.32 \pm 0.04$ in the Intermediate group, and $0.15 \pm 0.04$ in the Active group; $P<0.001$ ). The presence and numbers of disabilities decreased as physical activity increased.

\section{Comparison of exhaustion and falls by physical activity level}

The proportions of participants with exhaustion in the Inactive, Intermediate, and Active groups were $262(36.0 \%), 132(25.4 \%)$, and $77(21.2 \%)$, respectively $(P<0.001)$. The numbers of exhaustion (means \pm SE) were $0.55 \pm 0.03$ in the Inactive group, $0.37 \pm$ 0.03 in the Intermediate group, and $0.31 \pm 0.03$ in the Active group (Fig. $1 \mathrm{~B}, P<0.001$ ). The multivariate analysis showed similar results $(0.56 \pm 0.03$ in the Inactive group, $0.38 \pm 0.03$ in the Intermediate group, and $0.30 \pm 0.04$ in the Active group; $P<0.001$ ). The presence of exhaustion decreased as physical activity increased. The number of patients with exhaustion was higher in the Inactive group than in the other groups. The numbers of participants with a history of fall during the last 12 months were 149 (20.5\%) in the Inactive group, 88 (16.9\%) 


\section{Kidney Blood Pressure Research}
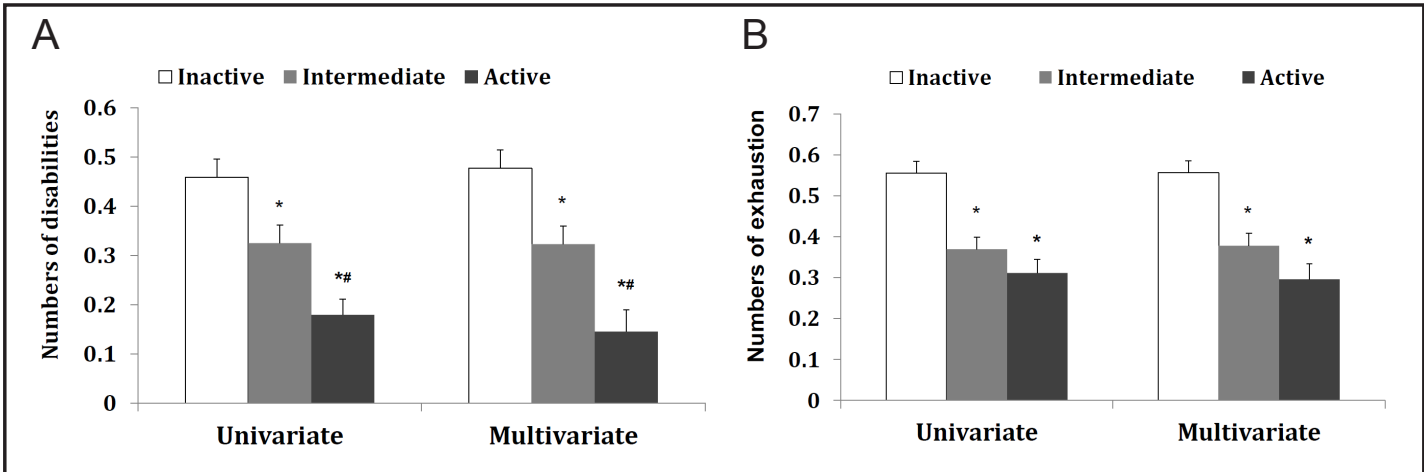

Fig. 1 Comparison of the numbers of disabilities (A) or exhaustion (B) among the groups by physical activity (data are expressed as mean and standard errors). (A) The number of disabilities (Univariate: $0.46 \pm 0.04$ in the Inactive group, $0.33 \pm 0.04$ in the Intermediate group, and $0.18 \pm 0.03$ in the Active group; Multivariate: $0.48 \pm 0.03$ in the Inactive group, $0.32 \pm 0.04$ in the Intermediate group, and $0.15 \pm 0.04$ in the Active group; $P<0.001$ for both analyses). (B) The number of exhaustion (Univariate: $0.55 \pm 0.03$ in the Inactive group, $0.37 \pm 0.03$ in the Intermediate group, and $0.31 \pm 0.03$ in the Active group; Multivariate: $0.56 \pm 0.03$ in the Inactive group, $0.38 \pm 0.03$ in the Intermediate group, and $0.30 \pm 0.04$ in the Active group; $P<0.001$ for both analyses). ${ }^{*} P<0.05$ versus the Inactive group. ${ }^{\#} P<0.05$ versus the Intermediate group

in the Intermediate group, and $48(13.2 \%)$ in the Active group $(P=0.011)$. The numbers of participants with history of serious falls included 27 (3.7\%) in the Inactive group, $11(2.1 \%)$ in the Intermediate group, and $9(2.5 \%)$ in the Active group $(P=0.219)$.

Comparison of HRQoL scale scores according to physical activity level

Table 3 shows the differences in HRQoL scale scores according to physical activity. On univariate analysis, PF, GH, VT, PCS, MCS, and QSI scores increased as physical activity increased. RP, BP, SF, MH, OHR, symptom/problems, kidney disease effects, kidney disease burden, cognitive function, sleep, social support, and dialysis staff encouragement scores were lower in the Inactive group than in the other groups. The mean RE score was higher in the Active group than in the Inactive group.

The SF-6 scale scores showed the same trends on multivariate analysis. The mean symptom/problems score was highest in the Active group. Scores for kidney disease burden, QSI, and dialysis staff encouragement were higher in the Active group than in the Inactive group. The social support score was higher in the Intermediate group than in the Inactive group.

Survival analysis by physical activity level

The survival rate for all-cause death was $99.7 \%$ at 100 days and $95.5 \%$ at 500 days in the Active group, $99.4 \%$ at 100 days and $95.2 \%$ at 500 days in the Intermediate group, and $99.9 \%$ at 100 days and $93.5 \%$ at 500 days in the Inactive group (Fig. $2 \mathrm{~A} ; P=0.232$ ). The survival rate for cardiovascular death was $99.7 \%$ at 100 days and $98.8 \%$ at 500 days in the Active group, $99.8 \%$ at 100 days and $98.3 \%$ at 500 days in the Intermediate group, and $100 \%$ at 100 days and $96.7 \%$ at 500 days in the Inactive group (Fig. $2 \mathrm{~B} ; P=0.064$ ).

\section{Discussion}

The present study was a multicenter cohort study and enrolled only relatively stable dialysis patients. The dialysis patients were divided into 3 groups by physical activity level. The most common cause of physical inactivity was unwillingness to exercise. The 


\section{Kidney Blood Pressure Research}

Table 3. Comparison of quality of life scale scores between hemodialysis and peritoneal dialysis patients

\begin{tabular}{|c|c|c|c|c|c|c|c|c|}
\hline \multirow[b]{2}{*}{ SF-6 scale } & \multicolumn{4}{|c|}{ Univariate (mean \pm SD) } & \multicolumn{4}{|c|}{ Multivariate $($ mean $\pm \mathrm{SE}$ ) } \\
\hline & Inactive & Intermediate & Active & $P$-value & Inactive & Intermediate & Active & $P$-value \\
\hline $\mathrm{PF}$ & $67.3 \pm 27.2$ & $77.4 \pm 20.4^{*}$ & $83.5 \pm 15.5^{* \#}$ & $<0.001$ & $67.0 \pm 0.7$ & $77.3 \pm 0.9^{*}$ & $84.4 \pm 1.0^{* \#}$ & $<0.001$ \\
\hline RP & $57.0 \pm 43.2$ & $68.7 \pm 39.9 *$ & $74.3 \pm 37.0 *$ & $<0.001$ & $57.1 \pm 1.5$ & $68.2 \pm 1.8^{*}$ & $74.8 \pm 2.1^{*}$ & $<0.001$ \\
\hline $\mathrm{BP}$ & $72.5 \pm 27.4$ & $80.3 \pm 22.3^{*}$ & $83.5 \pm 24.0^{*}$ & $<0.001$ & $72.9 \pm 0.9$ & $79.8 \pm 1.1^{*}$ & $83.3 \pm 1.3^{*}$ & $<0.001$ \\
\hline GH & $39.5 \pm 21.2$ & $46.5 \pm 22.1^{*}$ & $50.9 \pm 23.4^{* \#}$ & $<0.001$ & $39.5 \pm 0.8$ & $46.2 \pm 1.0^{*}$ & $51.2 \pm 1.2^{* \#}$ & $<0.001$ \\
\hline VT & $38.7 \pm 20.3$ & $47.3 \pm 19.9^{*}$ & $53.1 \pm 21.4^{* \#}$ & $<0.001$ & $38.7 \pm 0.7$ & $47.0 \pm 0.9^{*}$ & $53.5 \pm 1.1^{\text {*\# }}$ & $<0.001$ \\
\hline SF & $72.5 \pm 28.9$ & $76.5 \pm 26.1^{*}$ & $80.0 \pm 26.8^{*}$ & $<0.001$ & $72.1 \pm 1.0$ & $76.5 \pm 1.2^{*}$ & $80.8 \pm 1.4^{*}$ & $<0.001$ \\
\hline RE & $67.1 \pm 43.3$ & $72.8 \pm 40.2$ & $75.4 \pm 40.0^{*}$ & 0.003 & $67.2 \pm 1.5$ & $72.5 \pm 1.8$ & $75.7 \pm 2.2^{*}$ & 0.004 \\
\hline $\mathrm{MH}$ & $55.0 \pm 20.8$ & $61.0 \pm 19.6^{*}$ & $63.6 \pm 19.4^{*}$ & $<0.001$ & $55.0 \pm 0.7$ & $60.7 \pm$ & $63.9 \pm 1.1^{*}$ & $<0.001$ \\
\hline OHR & $32.4 \pm 26.2$ & $40.3 \pm 24$ & $43.5 \pm 25.7^{*}$ & $<0.001$ & $32.5 \pm 0.9$ & $40.0 \pm 1$ & $43.6 \pm 1.3^{*}$ & 001 \\
\hline PCS & $55.0 \pm 21.6$ & $64.0 \pm 18.3^{*}$ & $69.1 \pm 17.9^{* \#}$ & $<0.001$ & $55.0 \pm 0.7$ & $63.7 \pm$ & $69.4 \pm 1.0^{* \#}$ & $<0.001$ \\
\hline MCS & $54.5 \pm 20.6$ & $60.8 \pm 19.3^{*}$ & $64.6 \pm 20.2^{* \#}$ & $<0.001$ & $54.5 \pm 0.7$ & $60.6 \pm 0.9 *$ & $65.0 \pm 1.0$ *\# & $<0.001$ \\
\hline KD-sp & & & & & & & & \\
\hline Sx & $7.2 \pm 16.3$ & $81.7 \pm$ & $83.9 \pm 12.7^{*}$ & $<0.001$ & 80.6 & 82.3 & $88.1 \pm 1.4^{* \#}$ & $<0.001$ \\
\hline $\mathrm{KD}$ & $70.1 \pm 20.2$ & $74.5 \pm 1$ & $76.8 \pm 18.3^{*}$ & & $73.9 \pm 1.3$ & 73. & $78.4 \pm 1.8$ & 99 \\
\hline KD burden & $32.5 \pm 25.7$ & $36.5 \pm 26.7^{*}$ & $38.0 \pm 27.0^{*}$ & $<0.001$ & $36.2 \pm 2.0$ & 37.6 & $45.3 \pm 2.7^{*}$ & 0.023 \\
\hline Work status & $29.0 \pm 36.0$ & $27.7 \pm 36.6$ & $25.8 \pm 36.1$ & 0.381 & $42.3 \pm 3.0$ & $40.2 \pm 3.3$ & $37.8 \pm 4.2$ & 0.673 \\
\hline Cognitive function & $83.6 \pm 19.3$ & $87.8 \pm 15.3^{*}$ & $88.3 \pm 15.6^{*}$ & $<0.001$ & $88.3 \pm 1.1$ & $89.6 \pm$ & $91.8 \pm 1.5$ & 0.175 \\
\hline QSI & $72.3 \pm 22.7$ & $76.3 \pm 21.1^{*}$ & $80.1 \pm 20.0^{* \#}$ & $<0.001$ & $76.5 \pm 1.5$ & $78.0 \pm 1.7$ & $83.7 \pm 2.2^{*}$ & 0.024 \\
\hline Sexual function & $76.3 \pm 28.0$ & $76.8 \pm 25.6$ & $80.6 \pm 23.1$ & 0.427 & $75.3 \pm 2.0$ & $76.7 \pm 2.2$ & $82.7 \pm 2.8$ & 0.094 \\
\hline Sleep & $61.8 \pm 21.4$ & $65.0 \pm 20.7^{*}$ & $66.7 \pm 21.8^{*}$ & $<0.001$ & $65.5 \pm 1.5$ & $66.7 \pm 1.7$ & $71.5 \pm 2.2$ & 0.072 \\
\hline Social & $64.3 \pm 27.4$ & $70.3 \pm 26.2^{*}$ & $71.0 \pm 26.6^{*}$ & $<0.001$ & $64.3 \pm 2.0$ & $73.4 \pm 2.2^{*}$ & $72.0 \pm 2.8$ & 0.006 \\
\hline tisfaction & $64.5 \pm 23.1$ & $66.3 \pm 22.4$ & $67.7 \pm 22.7$ & 0.077 & $65.4 \pm 1.8$ & $67.5 \pm 2.0$ & $69.6 \pm 2.5$ & 0.392 \\
\hline DSE & $84.0 \pm 19.8$ & $87.4 \pm 17.7^{*}$ & $89.1 \pm 16.0 *$ & $<0.001$ & $84.8 \pm 1.4$ & $88.3 \pm 1.5$ & $90.5 \pm 1.9^{*}$ & 0.039 \\
\hline
\end{tabular}

Univariate analyses were tested by one way analysis of variance, followed by a post hoc Tukey comparison. Multivariable analyses were adjusted for age, sex, body mass index, education level, dialysis vintage, diabetes mellitus, cerebrovascular disease, coronary artery disease, serum albumin level, intact-parathyroid hormone level, and dialysis modality. $* P<0.05$ versus the Inactive group. $\# P<0.05$ versus the Intermediate group. Abbreviations: SD, standard deviation; SE, standard error; SF, short form; PF, physical functioning; RP, role limitations due to physical health problems; BP, bodily pain; GH, general health; VT, vitality; SF, social functioning; RE, role limitations due to emotional problems; $\mathrm{MH}$, mental health; OHR, overall health rating; PCS, physical component scale; MCS, mental component scale; KD, kidney disease; Sx, symptom/problems; QSI, quality of social interaction; DSE, dialysis staff encouragement

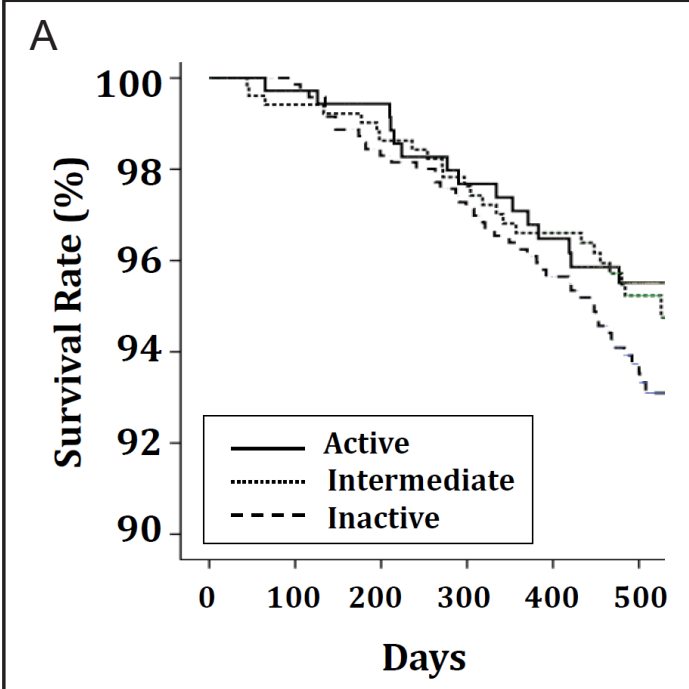

B

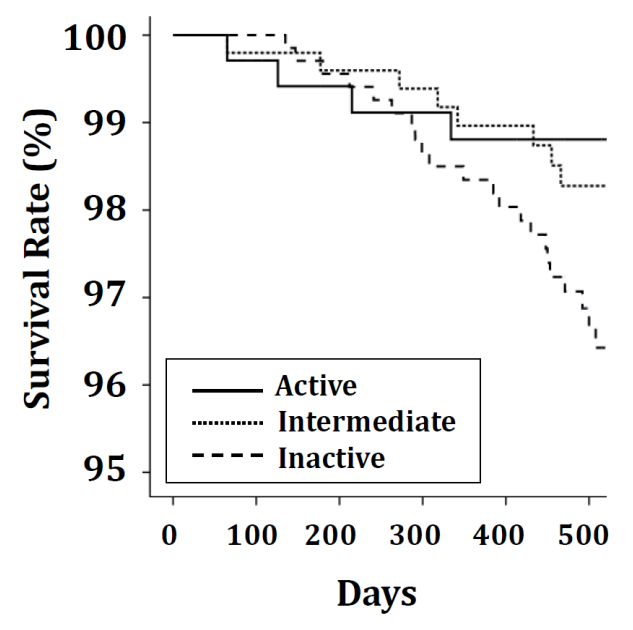

Fig. 2 Kaplan-Meier curves (A, all-cause mortality; B, cardiovascular mortality).

proportions of frailty, disability, and exhaustion decreased as physical activity increased. More participants in the Inactive group had a history of fall s during the last 12 months. Most of the HRQoL scale scores were highest in the Active group. No statistical significance was observed; however, the trend showed highest survival rates in the Active group.

Previous studies showed positive associations between physical activity and PCS, MCS, or survival in dialysis patients $[12,14,15,18]$. However, those studies mainly enrolled 


\section{Kidney Blood Pressure Research}

Western populations. Many factors, such as differences in disease burden, socioeconomic status, social support, or insurance, can influence HRQoL scale scores. Ethnicity per se is associated with differences in HRQoL results in dialysis patients [32, 33]. Therefore, further investigations are needed to identify the similarity or difference in the association between 2 variables in Korean dialysis patients compared with the results from Western dialysis patients. The present study enrolled only Korean dialysis patients. To the best of our knowledge, few studies have examined the association between physical activity and HRQoL in Korean dialysis patients. In addition, the present study enrolled only relatively stable dialysis patients and may have excluded the potential effects, such as acute medical or psychological problems, that can develop in incident dialysis patients. Our results revealed that physical activity was associated with most SF-36 components, including kidney disease burden, QSI, and dialysis staff encouragement. Our results were similar to those of previous studies. However, the survival analyses did not show statistical significance. The nonsignificance of the findings of the survival analyses would be associated with the relatively short follow-up period and low mortality rate owing to its enrollment of relatively stable dialysis patients. In addition, our study was a retrospective study. However, considering the intergroup differences in survival rate and Kaplan-Meier curve shapes, further studies with longer follow-up periods may reveal statistical significance in both all-cause and cardiovascular mortality rates.

There are no guidelines regarding the optimal time to evaluate HRQoL in dialysis patients, and only few studies are conducted. We believe that the optimal time to evaluate HRQoL in dialysis patients or the significant differences in HRQoL before, during, or after dialysis are very interesting issues. Most participants in our study were evaluated during dialysis sessions.

Our study investigated the cause of physical inactivity and found that the most common cause was unwillingness to exercise. Unwillingness to exercise, fatigue, lack of time to exercise, pain, unsure how to exercise, depressive mood, lack of equipment or place to exercise, and unsure about the importance of exercise may be reversible or resoluble. Of the total dialysis patients, $45.2 \%$ were included in the Inactive group; however, proper psychological or medical supports can be used to move participants from the Inactive group to the Active or Intermediate group. For example, lack of time, equipment, or place would be main a challenge of physical inactivity or exercise in dialysis patients; however, these may be overcame by exercise during dialysis sessions. Saitoh et al. have recently investigated the effects of intradialytic exercise in dialysis [34]. Their preliminary results showed that intradialytic exercise was safe and improved physical activity and malnutrition. Although further investigations are needed to identify the effects and safety of intradialytic exercise and establish protocols for exercise, intradialytic exercise may be an option to overcome physical inactivity or increase exercise in dialysis patients. In our study, the specific causes of unwillingness to exercise were not included. The purpose of our study was to evaluate the association between physical activity and various clinical outcomes in dialysis patients. Further analyses regarding specific causes of unwillingness, which were beyond the scope of our study, may be useful in future investigations.

Frailty is defined as a state of high vulnerability to adverse outcomes including disability and dependency combined with decreased body energy, protein reserve, and strength [35]. The association between physical activity and frailty is well-known. One review showed that the prevalence of frailty is approximately $24-78 \%$ in prevalent dialysis patients [36]. In our study of relatively stable dialysis patients, the prevalence of frailty was $34.6 \%$, and those in the Inactive and Active groups were $52.7 \%$ and $14.9 \%$, respectively. The prevalence of frailty in the total participants was similar to those of a previous report; however, there was a significant difference in this prevalence between the Inactive and Active groups.

Our study showed an association between physical activity and frailty or exhaustion. Frailty includes physical inactivity as a diagnostic criterion for frailty; thus, the comparison of physical activity and frailty may be duplicated. Endurance is a diagnostic criterion for 


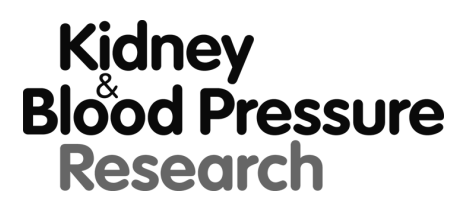

frailty and interchangeable with exhaustion. Therefore, we compared each component for frailty, including slowness, poor endurance, and unintentional weight loss. The proportion of participants with each component decreased as physical activity increased. In addition, considering the accuracy of the exhaustion definition, we defined exhaustion using another 2 questions drawn from the Center for Epidemiological Studies Depression Scale [29]. The Classical Center for Epidemiologic Studies Depression Scale (CESD) was created for evaluation of depressive symptoms and included a 20-item self-report measure [27]. Two items among the 20 items are associated with exhaustion [37]. Data on all items would be useful. However, we want to evaluate exhaustion rather than depression, and considering the limitations of time or effort, we have evaluated only the 2 items associated with exhaustion.

Disability is defined as difficulty or dependency in performing activities essential to independent living $[38,39]$. Classical disability measurements published by Katz et al. include 6 items [40]. However, the incontinence item was highly associated with urinary incontinence and age [41]. Many authors have used modified ADL scales, including meal, dress/undress, get in or out of bed, toileting, and bathing. However, not all 5 items were sometimes asked because of limitation of time or effort [42]. Some surveys have also combined 2 similar activities, such as toileting and bathing into 1 item [26]. Our study had evaluated disability using 4 items (meal, dress/undress, get in or out of bed, and take a bath or shower) among the 6 classical items. Although the reliability or validity for the 4 items was not investigated, we think that only 4 items would be sufficient to identify the disability considering limitations of time or effort. Disability is a status that includes both social and medical entities [38]. Disability can be a cause of frailty; however, frailty can also be a risk factor for the development of disability. Disability and frailty can overlap; however, these 2 clinical entities are distinct and can exist independently [43]. Disability per se can be associated with poor clinical outcomes. Therefore, we evaluated both disability and frailty. In addition, frailty, disability, or exhaustion can be conclusively associated with development of falls in dialysis patients [44]. In our study, the proportion of participants with a history of falls during the last 12 months increased as physical activity decreased. The highest proportion of participants with a history of serious falls was found in the Inactive group; however, no statistical significance was observed.

In our study, there were older participants and higher comorbidities in the Active group than the other groups. These would misinterpret older participants and those with high comorbidities to be more physically active. However, there were no significant differences in the proportions with CAD or CVA among the 3 groups. Although statistical significance in age was obtained, we think that the mean differences in age may be not enough to induce clinical effects. In addition, the Active group had higher proportions of men than the other groups. Sex differences among the 3 groups would influence physical activity and compensate for age effects.

The present study has a few limitations. First, it was retrospective study and used participants enrolled in a previous study [24]. Second, physical activity was assessed by using only a self-reported questionnaire. This subjective method may be associated with overestimation or underestimation of physical activity. Objective methods, such as accelerometer or physical activity monitor have more clinical importance or accuracy than our method. Unfortunately, our study did not include other methods and used only a selfreported questionnaire. This is an inherent limitation of our study. However, many previous studies have also assessed physical activity using this questionnaire; WHO also defined physical activity level using this questionnaire. This may be associated with frailty, disability, or exhaustion inaccuracies. Third, data on dialysis adequacy, use of opioid, antihistamine, or antidepressant, and residual renal function were not included. We did not compare physical activity with changes in dialysis dose nor evaluate the difference in dialysis dose among the 3 groups by physical activity. Whether the dialysis of the participants was adequate or not can be associated with physical activity. KDOQI recommend $s p K t / V \geq 1.2$ as a minimally adequate dialysis for HD [45]. In our study, most common dialysis modality was HD. From the 2015 


\section{Kidney \\ Blood Pressure Research}

Korean Registry data, spKt/V in men and women who underwent HD was $1.43 \pm 0.25$ and $1.71 \pm 0.29$, respectively [46]. Therefore, most Korean dialysis patients, including the patients in our study, may undergo adequate dialysis. In addition, use of opioid, antihistamine, or antidepressant can be associated with decreased physical activity and cognitive function. Although our study did not evaluate these medications, all participants in our study were relatively stable dialysis patients and able to walk and communicate with the interviewer. We think that the number of participants significantly affected by these medications would be small. Fourth, the associations between physical activity and variables, including HRQoL, frailty, exhaustion, and disability were evaluated using data collected at enrollment. Analysis of the changes between data at enrollment and those at follow-up may accurately identify the associations better. A prospective study including additional parameters such as other physical performance-based measurements and follow-up data is warranted to overcome these limitations.

\section{Conclusion}

In conclusion, high physical activity was associated with favorable results for most HRQoL scale scores, including frailty, disability, and exhaustion, in Korean dialysis patients. Patients on dialysis should be encouraged to increase their physical activity, which may improve their prognosis.

\section{Disclosure Statement}

The authors declare that they have no conflict of interest.

\section{Acknowledgements}

Thisworkwas supported by the Medical Research Center Program(2015R1A5A2009124) through the National Research Foundation of Korea (NRF) funded by the Ministry of Science, ICT and Future Planning.

\section{References}

1 US Renal Data System, USRDS 2015 Annual Data Report: Atlas of Chronic Kidney Disease in the United States, National Institutes of Health, National Institute of Diabetes and Digestive and Kidney Diseases, Bethesda, MD, 2015.

2 Jin DC: Current Renal Replacement Therapy in Korea. Proceedings of the $35^{\text {th }}$ Annual Spring Meeting of the Korean Society of Nephrology. 2015;Suppl1:117-136.

-3 Jofre R, Lopez-Gomez JM, Moreno F, Sanz-Guajardo D, Valderrabano F: Changes in quality of life after renal transplantation. Am J Kidney Dis 1998;32:93-100.

-4 Purnell TS, Auguste P, Crews DC, Lamprea-Montealegre J, Olufade T, Greer R, Ephraim P, Sheu J, Kostecki D, Powe NR, Rabb H, Jaar B, Boulware LE: Comparison of life participation activities among adults treated by hemodialysis, peritoneal dialysis, and kidney transplantation: a systematic review. Am J Kidney Dis 2013;62:953-973.

-5 Grzegorzewska AE, Izdebska A, Niepolski L, Warchoł W, Jagodziński PP: Self-Reported Physical Activity, Quality of Life, and Psychological Status in Relation to Plasma 25-Hydroxyvitamin D Concentration in Patients Treated with Hemodialysis. Kidney Blood Press Res 2016;41:886-900.

6 Buckner S, Dwyer J: Do we need a nutrition-specific quality of life questionnaire for dialysis patients? J Ren Nutr 2003;13:295-302. 


\section{Kidney \\ Blood Pressure Research}

7 Germin-Petrović D, Mesaros-Devcić I, Lesac A, Mandić M, Soldatić M, Vezmar D, Petrić D, Vujicić B, BasićJukić N, Racki S: Health-related quality of life in the patients on maintenance hemodialysis: the analysis of demographic and clinical factors. Coll Antropol 2011;35:687-693.

-8 Wang V, Seow YY, Chow WL: Influence of ethnicity on health related quality of life of hemodialysis patients in Singapore. Int J Artif Organs 2012;35:217-225.

9 World Health Organization: Global recommendations on physical activity for health. Geneva, Switzerland: World Health Organization; 2010.

10 Kielstein H, Suntharalingam M, Perthel R, Song R, Schneider SM, Martens-Lobenhoffer J, Jäger K, BodeBöger SM, Kielstein JT: Role of the endogenous nitric oxide inhibitor asymmetric dimethylarginine (ADMA) and brain-derived neurotrophic factor (BDNF) in depression and behavioural changes: clinical and preclinical data in chronic kidney disease. Nephrol Dial Transplant 2015;30:1699-1705.

11 Barcellos FC, Santos IS, Umpierre D, Bohlke M, Hallal PC: Effects of exercise in the whole spectrum of chronic kidney disease: a systematic review. Clin Kidney J 2015;8:753-765.

12 Painter P, Carlson L, Carey S, Paul SM, Myll J: Physical functioning and health-related quality-of-life changes with exercise training in hemodialysis patients. Am J Kidney Dis 2000;35:482-492.

13 O’Hare AM, Tawney K, Bacchetti P, Johansen KL: Decreased survival among sedentary patients undergoing dialysis: Results from the dialysis morbidity and mortality study wave 2. Am J Kidney Dis 2003;41:447-454.

14 Stack AG, Molony DA, Rives T, Tyson J, Murthy BV: Association of physical activity with mortality in the US dialysis population. Am J Kidney Dis 2005;45:690-701.

-15 Tentori F, Elder SJ, Thumma J, Pisoni RL, Bommer J, Fissell RB, Fukuhara S, Jadoul M, Keen ML, Saran R, Ramirez SP, Robinson BM: Physical exercise among participants in the Dialysis Outcomes and Practice Patterns Study (DOPPS): Correlates and associated outcomes. Nephrol Dial Transplant 2010;25:30503062.

16 Heiwe S, Jacobson SH: Exercise training for adults with chronic kidney disease. Cochrane Database Syst Rev 2011;5:CD003236.

17 Painter P, Marcus RL: Assessing physical function and physical activity in patients with CKD. Clin J Am Soc Nephrol 2013;8:861-872.

18 Johansen KL, Kaysen GA, Dalrymple LS, Grimes BA, Glidden DV, Anand S, Chertow GM: Association of physical activity with survival among ambulatory patients on dialysis: The Comprehensive Dialysis Study. Clin J Am Soc Nephrol 2013;8:248-253.

19 Painter P, Roshanravan B: The association of physical activity and physical function with clinical outcomes in adults with chronic kidney disease. Curr Opin Nephrol Hypertens 2013;22:615-623.

20 Lopes AA, Lantz B, Morgenstern H, Wang M, Bieber BA, Gillespie BW, Li Y, Painter P, Jacobson SH, Rayner HC, Mapes DL, Vanholder RC, Hasegawa T, Robinson BM, Pisoni RL: Associations of self-reported physical activity types and levels with quality of life, depression symptoms, and mortality in hemodialysis patients: the DOPPS. Clin J Am Soc Nephrol 2014;9:1702-1712.

21 Matsuzawa R, Matsunaga A, Wang G, Kutsuna T, Ishii A, Abe Y, Takagi Y, Yoshida A, Takahira N: Habitual physical activity measured by accelerometer and survival in maintenance hemodialysis patients. Clin J Am Soc Nephrol 2012;7:2010-2016.

-22 Dziubek W, Kowalska J, Kusztal M, Rogowki Ł, Gołębiowski T, Nikifur M, Szczepańska-Gieracha J, ZembrońŁacny A, Klinger M, Woźniewski M: The Level of Anxiety and Depression in Dialysis Patients Undertaking Regular Physical Exercise Training--a Preliminary Study. Kidney Blood Press Res 2016;41:86-98.

23 Dungey M, Bishop NC, Young HM, Burton JO, Smith AC: The Impact of Exercising During Haemodialysis on Blood Pressure, Markers of Cardiac Injury and Systemic Inflammation--Preliminary Results of a Pilot Study. Kidney Blood Press Res 2015;40:593-604.

24 Lee SY, Kim JC, Yang DH, Hwang EA, Park JW, Park SH, Kim TW, Lee DH, Park KS: Frailty as an independent predictor for hospitalization-free survival even in relatively healthy patients receiving chronic dialysis. Proceedings of the $35^{\text {th }}$ Annual Spring Meeting of the Korean Society of Nephrology. 2015;Suppl1:215.

25 Park HJ, Kim S, Yong JS, Han SS, Yang DH, Meguro M, Han CW, Kohzuki M: Reliability and validity of the Korean version of Kidney Disease Quality of Life instrument (KDQOL-SF). Tohoku J Exp Med 2007;211:321329.

26 Ware JE Jr, Sherbourne CD: The MOS 36-item short-form health survey (SF-36). I. Conceptual framework and item selection. Med Care 1992;30:473-483. 


\section{Kidney \\ Blood Pressure Research}

Kang et al.: Physical Activity in Dialysis Patients

27 McHorney CA, Ware JE Jr, Raczek AE: The MOS 36-item short-form health survey (SF-36): II. Psychometric and clinical tests of validity in measuring physical and mental health constructs. Med Care 1993;31:247263.

28 Woods NF, LaCroix AZ, Gray SL, Aragaki A, Cochrane BB, Brunner RL, Masaki K, Murray A, Newman AB; Women's Health Initiative: Frailty: emergence and consequences in women aged 65 and older in the Women's Health Initiative Observational Study. J Am Geriatr Soc 2005;53:1321-1330.

29 Orme J, Reis J, Herz E: Factorial and discriminate validity of the Center for Epidemiological Studies Depression (CES-D) Scale. J Clin Psychol 1986;42:28-33.

-30 Buchner DM, Hornbrook MC, Kuntner NG, Tinetti ME, Ory MG, Mulrow CD, Schechtman KB, Gerety MB, Fiatarone MA, Wolf SL, Rossiter J, Arfken C, Kanten K, Lipsitz LA, Sattin RW, DeNino LA and FICSIT Group: Development of the common data base for the FICSIT trials. J Am Geriatr Soc 1993;41:297-308.

-31 Johansen KL, Chertow GM, Jin C, Kutner NG: Significance of frailty among dialysis patients. J Am Soc Nephrol 2007;18:2960-2967.

-32 Bakewell AB, Higgins RM, Edmunds ME: Does ethnicity influence perceived quality of life of patients on dialysis and following renal transplant? Nephrol Dial Transplant 2001;16:1395-1401.

33 Unruh M, Miskulin D, Yan G, Hays RD, Benz R, Kusek JW, Meyer KB; HEMO Study Group: Racial differences in health-related quality of life among hemodialysis patients. Kidney Int 2004;65:1482-1491.

34 Saitoh M, Ogawa M, Rodrigues Dos Santos M, Kondo H, Suga K, Itoh H, Tabata Y: Effects of Intradialytic Resistance Exercise on Protein Energy Wasting, Physical Performance and Physical Activity Ambulatory Patients on Dialysis: A Single-Center Preliminary Study in a Japanese Dialysis Facility. Ther Apher Dial 2016;20:632-638.

-35 Wilhelm-Leen ER, Hall YN, K Tamura M, Chertow GM: Frailty and chronic kidney disease: the Third National Health and Nutrition Evaluation Survey. Am J Med 2009;122:664-671.

-36 Johansen KL: The Frail Dialysis Population: A Growing Burden for the Dialysis Community. Blood Purif 2015;40:288-292.

-37 Fried LP, Tangen CM, Walston J, Newman AB, Hirsch C, Gottdiener J, Seeman T, Tracy R, Kop WJ, Burke G, McBurnie MA; Cardiovascular Health Study Collaborative Research Group: Frailty in older adults: evidence for a phenotype. J Gerontol A Biol Sci Med Sci 2001;56:M146-156.

38 Nagi SZ: Disability concepts revisited: implications for prevention; in AM Pope, AR Tarlov (eds): Disability in America: Toward a National Agenda for Prevention. Washington, DC, National Academy Press, 1980, pp. 309-327.

-39 Adams PF, Hendershot GE, Marano MA: Current Estimates from the National Health Interview Survey, 1996. Vital Health Stat 1999;10:1-203.

40 Katz S, Downs TD, Cash HR, Grotz RC: Progress in development of the index of ADL. Gerontologist 1970;10:20-30.

41 Tufan F, Topcu Y, Bahat G, Kara MA: Limitations of the Continence Item in Katz Activities of Daily Living Scale. Gerontol Geriatr Res 2016;2:1008.

42 Wiener JM, Hanley RJ, Clark R, Van Nostrand JF: Measuring the activities of daily living: comparisons across national surveys. J Gerontol 1990;45:S229-237.

-43 Fried LP, Ferrucci L, Darer J, Williamson JD, Anderson G: Untangling the concepts of disability, frailty, and comorbidity: implications for improved targeting and care. J Gerontol A Biol Sci Med Sci 2004;59:255-263.

-44 Delgado C, Shieh S, Grimes B, Chertow GM, Dalrymple LS, Kaysen GA, Kornak J, Johansen KL: Association of Self-Reported Frailty with Falls and Fractures among Patients New to Dialysis. Am J Nephrol 2015;42:134140.

45 National Kidney Foundation: KDOQI Clinical Practice Guidelines and Clinical Practice Recommendations for 2006 Updates: Hemodialysis Adequacy, Peritoneal Dialysis Adequacy and Vascular Access. Am J Kidney Dis 2006;48:S1-322.

46 ESRD Registry Committee, Korean Society of Nephrology: Current renal replacement therapy in Korea 2016. Cited 10 April 2017. Available at: http://www.ksn.or.kr/file/sinchart/982988834_eddaa4e5_2016_ EC9B90EA B3A0.pdf . 